\title{
Optimisation of hydraulic fracture placement design in anisotropic shale reservoirs using azimuthal LWD sonic and spectral gamma ray analysis.
}

\author{
OLUYEMI, G.F. and AFOLABI, T.S.
}




\title{
OPTIMIZATION OF HYDRAULIC FRACTURE PLACEMENT DESIGN IN ANISOTROPIC SHALE RESERVOIRS USING AZIMUTHAL LWD SONIC AND SPECTRAL GAMMA RAY ANALYSIS
}

\section{Gbenga Folorunso Oluyemi ${ }^{1 *}$ and Tomilayo Simisola Afolabi School of Engineering, Robert Gordon University; AB10 7GJ Aberdeen UK}

\author{
${ }^{1}$ Tel: +44122426242; email: g.f.oluyemi@rgu.ac.uk \\ 2Tel: +44122426242; email: t.s.afolabi@rgu.ac.uk
}

\section{*Corresponding author}

\begin{abstract}
Hydraulic fracturing (fracking) of shale resources has become a trend in the oil and gas industry that is associated with exorbitant costs. This calls for certainty in the productivity and success of every fracture stimulation program. Current fracking practices rely mostly on a geometric design and evaluation of vertical well sections and pilot holes to predict properties along wellbore laterals. Consequently, there is a reduction in the efficiency of fracture stimulation programs and productivity of shale reservoirs. This problem is associated with the fact that shale reservoirs are anisotropic, possessing directional properties that cannot be accurately predicted as such.

In order to increase the efficiency of fracture stimulation, considerations have to be given to the anisotropic tendencies of shale petrophysical and geomechanical properties along wellbore laterals where fractures are hydraulically induced. In this study, an approach for the accurate quantification of vertical transverse isotropy (VTI) and resultant anisotropic properties along shale wellbore laterals using an LWD azimuthal sonic log was investigated.

Using the case study of a Marcellus shale well in Northeastern Pennsylvania having azimuthal sonic data, a work flow for obtaining anisotropic properties, critical to fracture stimulation design was developed. An algorithm for the characterization of wellbore geomechanical quality based on estimated VTI anisotropy, anisotropic closure stress and brittleness was also developed.
\end{abstract}

Keywords: fracking, vertical transverse anisotropy, stimulation, shale resources, geomechanical properties, petrophysical properties

Reference to this paper should be made as follows:

Oluyemi, G. F. and Afolabi, T.S. Optimisation of Hydraulic Fracture Placement Design in Anisotropic Shale Reservoirs Using Azimuthal LWD Sonic and Spectral Gamma Ray Analysis. Int. J. Petroleum Engineering

\section{Biographical Notes:}

Gbenga Oluyemi is a Lecturer at the School of Engineering, Robert Gordon University, Aberdeen. His research interests cover areas such as rock geomechanics, oilfield chemistry, flow assurance and uncertainty analysis. $\mathrm{He}$ is a chartered engineer in the UK and a Fellow of the UK HEA, He holds a PhD degree in Petroleum Geomechanics.

Tomilayo Afolabi is a Petroleum Engineer and holds an MSc from Robert Gordon University, Aberdeen. Her research interests is in fracture geomechanics. 


\subsection{INTRODUCTION}

Over the past decade there has been focus on the exploration and production of shale resources in North America. These unconventional resources represent a promising source of energy in several other countries including the United Kingdom, China, Saudi Arabia and India (Mickael, Barnett and Diab 2012). The two major technologies employed in the production of such formations are the drilling of horizontal wells and hydraulic fracturing. In effect, commercial viability has a direct dependence on these operations.

An effective hydraulic fracture placement is one that provides maximum fluid conductivity and ensures wellbore stability through optimal number of fracture stages, propagation, geometry and maintenance of fracture geometry through the productive life of the reservoir. This requires zonal characterisation to obtain the petrophysical and geomechanical properties of the wellbore.

One major attribute of shale reservoirs that results in uncertainty in hydraulic fracture stimulation design is anisotropy. This is the directional variability in the permeability, rock strength and in-situ stresses around and along the wellbore. Two types of anisotropy are commonly observed in shale reservoirs; these are HTI (Horizontal Transverse Isotropy) and VTI (Vertical Transverse Isotropy). Adequate consideration of these two forms of anisotropy in the design of fracture placement will result in effectiveness and optimum productivity. The HTI anisotropy is usually stress induced and commonly available from traditional monopole log data gathered in the vertical section of the well.

However, the VTI anisotropy, which is intrinsic and due to the horizontal layering in shale, is commonly neglected in the design of fracture placement (Mickael, Barnett and Diab 2012). This is due to the complexities and risks involved in evaluation and characterization of the properties in horizontal laterals. Thus, there is usually an insufficiency of information on anisotropic properties in horizontal wellbores and uncertainty in geomechanical characterisation.

This has led to the use of traditional geometric designs for the placement of fractures in most wells (Amorocho, Langford and Mejia 2014, and Kennedy et al. 2012). However, studies of production data from wells have shown less than average productivity from such wells (Kennedy et al. 2012, Andrew and Anthony 2014).

As a result, there is a demand for routine access to VTI anisotropy evaluation and well-specific geomechanical models in horizontal wellbores as erroneous fracture placement due to insufficiency of information leads to excessive well costs and reduced productivity. This is in direct response to the current quest in the industry for the reduction of uncertainty in fracture placement design and ultimately production optimization of shale reservoirs.

In this work, the role of anisotropic geomechanical properties in the optimisation of hydraulic fracture placement in shale reservoirs is presented. An effective optimisation approach for hydraulic fracture placement in anisotropic shale is also developed.

\subsection{MATERIALS AND METHODS}

A logging suite, containing azimuthal LWD sonic and spectral gamma ray plots from an horizontal Marcellus shale well, was used for the analysis carried out in this study. The log is presented in figure 1.0.

The obtained images were digitized using the Grapher $11^{\odot}$ software, followed by petrophysical and geomechanical evaluation using Microsoft Excel $^{\odot}$. The geomechanical evaluation involved the computation of directional elastic stiffness constants, VTI anisotropy, poisson's ratio, young's modulus, closure stress and brittleness while the petrophysical evaluation involved estimation of the Total Organic Content (TOC), porosity and clay mineral content. The elastic moduli approach was used to obtain a matrix of elastic stiffness constants from which other geomechanical properties were defined.

Subsequently, the petrophysical and geomechanical evaluation were integrated with an algorithm developed for the grouping of similar properties along the wellbore. This was used to obtain an optimal number of fracture 
stages, with location in the well such that a complex fracture network was successfully created in the productive zones along the wellbore.

\subsection{PETROPHYSICAL EVALUATION}

Petrophysical evaluation involved the acquisition of properties relating to the interaction of rock matrix and fluid. The first task was the estimation of TOC (Total Organic Carbon) along the wellbore lateral using a typical correlation of Uranium content and TOC for Marcellus shale. The cross plot between TOC and Uranium content is shown in Figure 2.0; the model fitted into the plot is presented in Equation 1.0.

$\mathrm{TOC}=$ Antilog $[0.0189 \mathrm{U}+0.336]$

Porosity was also estimated using the average of the up, down, left and right compressional wave signatures shown in track 4 of Figure 1.0 .

This is due to the fact that compressional waves are more representative of the formation as they travel through both the rock matrix and the fluid; in contrast shear wave travels mainly through the matrix. Different correlations between compressional wave transit time and porosity were used based on lithology. In the limestone and shaly carbonate zones, Wyllie's Time average equation (developed specifically for sandstones and limestone) was used as shown in Equation (2.0) (Malcolm 2000). In the shale regions, a correlation specific to shale reservoirs was used as proposed in Malcolm (2000) and Paul (2012). This is shown in Equation (3.0).

$\varphi($ limestone $)=\frac{\operatorname{ITT}(\log )-\operatorname{ITT}(\mathrm{m})}{\operatorname{ITT}(\mathrm{F})-\operatorname{ITT}(\mathrm{m})}$

$\varphi($ shale $)=\frac{I T T(\log )-I T T(m)}{214.6}$

Where;

$\varphi=$ porosity

ITT $(\log )=$ Interval Transit Time obtained from log.

$\operatorname{ITT}(\mathrm{m})=$ Interval Transit Time for rock matrix. A value of $62.5 \mu \mathrm{s} / \mathrm{ft}$ was used for the shale regions and $47.6 \mu \mathrm{s} / \mathrm{ft}$ for limestone regions (Carmichael 1982).

ITT(F) = Interval Transit Time for formation fluid. A value of $400 \mu \mathrm{s} / \mathrm{ft}$ was used in this formation, which is an average of the transit time of $70 \%$ gas and $30 \%$ water saturation associated with Marcellus shale (Taylor 2014). A gas correction factor of 0.7 was used in the wyllie's equation (Paul 2012).

\subsection{GEOMECHANICAL EVALUATION}

Geomechanical evaluation was carried out using the data obtained from the azimuthal sonic log signatures. Hence, the geomechanical properties acquired along the wellbore lateral were directional and based on VTI anisotropy. The azimuthal LWD sonic tool is able to differentiate the slowness of compressional and shear waves in different azimuthal directions around the borehole and sort the slowness in 16 sectors. This generates 16 azimuthal DTC and DTS curves which were reduced to up, right, down and left quadrants shown in tracks 4 and 5 of Figure 1.0, and further reduced to horizontal and vertical directions in this study.

A good agreement is observed between the four directional DTC curves. This is due to the fact that compressional waves do not undergo polarization (splitting in various directions) and are therefore transmitted from the tool parallel to the borehole. So, the four DTC quadrants correspond to the horizontal P-waves velocities only and cannot be solely used to quantify VTI anisotropy. 
However, shear waves undergo polarization in anisotropic medium and are transmitted in two directions, which is the reason for the significant discrepancy in the DTS quadrants shown in track 5 of Figure 1.0. Hence, VTI anisotropy was quantified using the shear wave signatures in the horizontal section of the well.

The basic sonic log workflow used in this study is shown in Figure 3.0.

Figure 3.0 shows that the elastic stiffness constants along the wellbore lateral were directly obtained from the sonic log based on the assumption of a VTI medium. The elastic constants are defined as follows for a VTI medium:

$\mathrm{C}_{11}$ is the elastic compressional stiffness coefficient in the horizontal plane transverse to the wellbore direction.

$\mathrm{C}_{22}$ is the elastic compressional stiffness coefficient in the horizontal plane in the direction of the wellbore.

$\mathrm{C}_{33}$ is the elastic compressional stiffness coefficient in the vertical plane.

$\mathrm{C}_{12}$ and $\mathrm{C}_{13}$ are elastic stiffness coefficient on the principal horizontal plane but with transverse directions.

$\mathrm{C}_{44}$ is the elastic slow shear stiffness coefficient in the vertical plane.

$C_{55}$ is the elastic fast shear stiffness coefficient in the vertical plane.

$\mathrm{C}_{66}$ is the elastic shear stiffness coefficient in the horizontal plane.

The equations for the estimation of the elastic constants are shown in Figure 4.0. Values of $\mathrm{C}_{44}$ and $\mathrm{C}_{55}$ shows good agreement (equal fast and slow shear in the horizontal direction). Therefore, the assumption of VTI anisotropy is justified.

Subsequently, other directional geomechanical properties such as VTI anisotropy, young's modulus, poisson's ratio and brittleness, were obtained using the equations shown in Table 1.0.

\subsection{INTEGRATION OF PETROPHYSICAL AND GEOMECHANICAL EVALUATION INTO HYDRAULIC FRACKING DESIGN}

In this study, an algorithm for the integration of well quality evaluation into fracking design for efficient selection of hydraulic fracturing intervals was developed and applied to the case study. The integration was carried out manually with the aid of Microsoft Excel. The algorithm, shown in Figure 5.0, is based on the methodology proposed in Ramakrishnan et al. 2009 where similar properties were grouped in fracking stages. The goal is to divide the borehole interval into zones that have comparable closure stresses such that one closure stress value can be representative of the whole zone. Zones with high porosity, high brittleness, high TOC, low closure stress and low anisotropy are desirable for fracking.

The petrophysical quality (PQ) along the wellbore lateral was classified as either desirable or undesirable based on a combination of TOC and porosity. Note that the terms 'desirable' or 'non-desirable' PQ is relative. A TOC threshold of $7 \%$ and porosity threshold of $3.0 \%$ were used in the case study.

The geomechanical quality (GQ) along the wellbore lateral were also classified as either 'desirable' or 'nondesirable' mostly based on the intersection of brittleness and closure gradient. The effect of anisotropy was included in the closure stress analysis as the closure stress is higher in regions with high anisotropy and lower in regions with low anisotropy. Brittleness was plotted on the scale of $0-100 \%$ while closure gradient on a scale of 0.5 to $0.9 \mathrm{psi} / \mathrm{ft}$. Regions on the scale where closure gradient was higher than brittleness were classified 'nondesirable' and vice versa. Depending on the petrophysical quality, the geomechanically non-desirable regions were either not considered in the hydraulic fracture design or required an improvement in design (such as reduction of cluster spacing) so as to attain the same level of efficiency as the other zones.

The geomechanical and petrophysical quality were combined for delineation of the wellbore into 'most suitable', 'less suitable' and 'least suitable' zones for fracking. The wellbore was then divided into segments based on lithology and each segment divided into fracking intervals. This division was based on a combination of the predetermined average stage spacing, minimum in-situ stresses and the pre-determined PQ and GQ. 


\section{DISCUSSION OF RESULTS}

The result of anisotropic and isotropic closure stress computation is shown in Figure 6.0. It is observed that the non-consideration of intrinsic anisotropy in the evaluation of closure stress yields $30-40 \%$ reduction in closure stress in the shale regions where VTI anisotropy is significantly high. 
The result of direct computation of VTI anisotropy is shown in track 2 of Figure 7.0. It is observed that the zone identified as shale (C) exhibits anisotropy in the range between $40 \%$ and $55 \%$, while the zone identified as limestone (D) exhibits anisotropy of approximately $7 \%$ (which is essentially isotropic). The anisotropy in the zone $\mathrm{C}$ is explained by the platy nature of the clay minerals abundant in shale, causing laminations and fissility. This causes intrinsic anisotropy in shale and a resulting difference between the horizontal and vertical properties. On the other hand, pure carbonate (zone D) formations are essentially homogenous and do not exhibit intrinsic anisotropy due to reduced clay mineral content.

The result of directional quantification of brittleness (shown in track 3 of Figure 7.0) yields higher values in the horizontal direction in the shale zone due to horizontal layering and large surface area per unit volume of the abundant clay minerals in the shale formations. Contrary to general belief, the results also show that shale possesses high brittleness values comparable with those in limestone formations in certain directions. This directional information can be used to optimize fracturing design.

The result of integration of the characterization in this study and hydraulic fracking design for the case study is shown in Figure 8.0 where there is a direct comparison with the conventional geometric design, that divides the wellbore lateral into an equal number of stages (with little or no consideration of anisotropy and lateral characterization). The matrix in Figure 9.0 was used to delineate the wellbore petrophysical, geomechanical and combined quality. The result shows that characterization work flow in this study yields a lower number of fracking stages and maximum efficiency.

Using the conventional geometric design, poor knowledge of the zones and grouping of dissimilar properties resulted in reduced efficiency as 4 out of 12 stages are poorly designed. As shown stage 2 possesses poor petrophysical quality and will not contribute to reservoir productivity. Stages 7, 9 and 11 show dissimilarity in properties such that stimulation could either result in borehole washout or insignificant fracture propagation.

On the other hand, the developed lateral characterization approach gives a good knowledge of the various zones. Therefore, stages 2, 8 and 11 will not be stimulated due to poor wellbore quality across the intervals. This leaves 8 stages with similar closure pressures across the interval in each stage. Hence, a resultant optimum reservoir drainage as all productive stages will be fracked and all perforations in each interval will be successfully fractured due to similar closure pressure across each interval.

\section{CONCLUSION}

An approach for the accurate quantification of vertical transverse isotropy (VTI) and resultant anisotropic properties in a Marcellus shale well using an LWD azimuthal sonic log was investigated. An algorithm for the characterization of wellbore geomechanical quality based on estimated VTI anisotropy, anisotropic closure stress and brittleness was also developed.

The study shows that:

- Shear slowness data obtained from the LWD azimuthal sonic log is critical to the quantification of VTI anisotropy and anisotropic shale property such as poisson's ratio, young's modulus, brittleness and closure stress.

- The estimation of closure stress without giving considerations to anisotropy in shale reservoirs will yield lower closure stress values. This will result in poor hydraulic fracture design as fractures may not attain breakdown or propagation.

- The use of the workflow in this study results in a more defined approach to delineating brittle/non-brittle zones unlike when clay content is used.

- Contrary to general belief, shale possesses higher brittleness values in certain directions that are comparable with the higher brittleness values in limestone formations.

- The lateral characterization approach to fracture placement design results in a more efficient design than the conventional geometric placement design.

- This approach reduces the need for drilling of vertical pilot holes for wellbore characterization. 


\section{REFERENCES}

1. AMOROCHO, C., LANGFORD, C. and MEJIA, C., 2014. Real Time Fracture Placement and Completion Design Using an Azimuthal LWD Sonic and Spectral Gamma Ray Analysis for an Anisotropic Unconventional Reservoir Evaluation. Society of Petroleum Engineers.

2. ANDREW P. B and ANTHONY P. P, 2014. Numerical Simulation of Simultaneous Growth of Multiple Interacting Hydraulic Fractures from Horizontal Wells. ProQuest ebrary. In: C.L. MEEHAN, J.M. VANBRIESEN and F. VAHEDIFARD, eds. Shale Energy Engineering 2014: Technical Challenges, Environmental Issues, and Public Policy. VA, USA: ASCE. pp. 201-211

3. CARMICHAEL, R.S., 1982. Handbook of Physical Properties of Rocks. Boca Raton, Florida: CRC Press Inc. 2, pp. 1-228.

4. KENNEDY, R.L. et al., 2012. Optimized Shale Resource Development: Proper Placement of Wells and Hydraulic Fracture Stages. Society of Petroleum Engineers.

5. MALCOLM R., 2002. The Geological interpretation of Well Logs. 2nd ed. Scotland: Rider-French Consulting LTD.

6. MICKAEL, M., BARNETT, C. and DIAB, M., 2012. Azimuthally Focused LWD Sonic Logging for Shear Wave Anisotropy Measurement and Borehole Imaging. Society of Petroleum Engineers.

7. PAUL G., 2012. Introduction to Petrophysics and Formation Evaluation. Leeds University.

8. RAMAKRISHNAN, $\mathrm{H}$. et al., 2009. Application of Downhole Injection Stress Testing in the Barnett Shale Formation. Society of Petroleum Engineers.

9. TAYLOR T., 2014. Lithostratigraphic and petrophysical analysis of the middle devonian marcellus shale at the mamont prospect, westmoreland county, pennsylvania [online] Clemson University, USA: Tiger Prints. Available from: http://tigerprints.clemson.edu/cgi/viewcontent.cgi?article $=2775 \&$ context $=$ all theses [Accessed July/20 2015] 


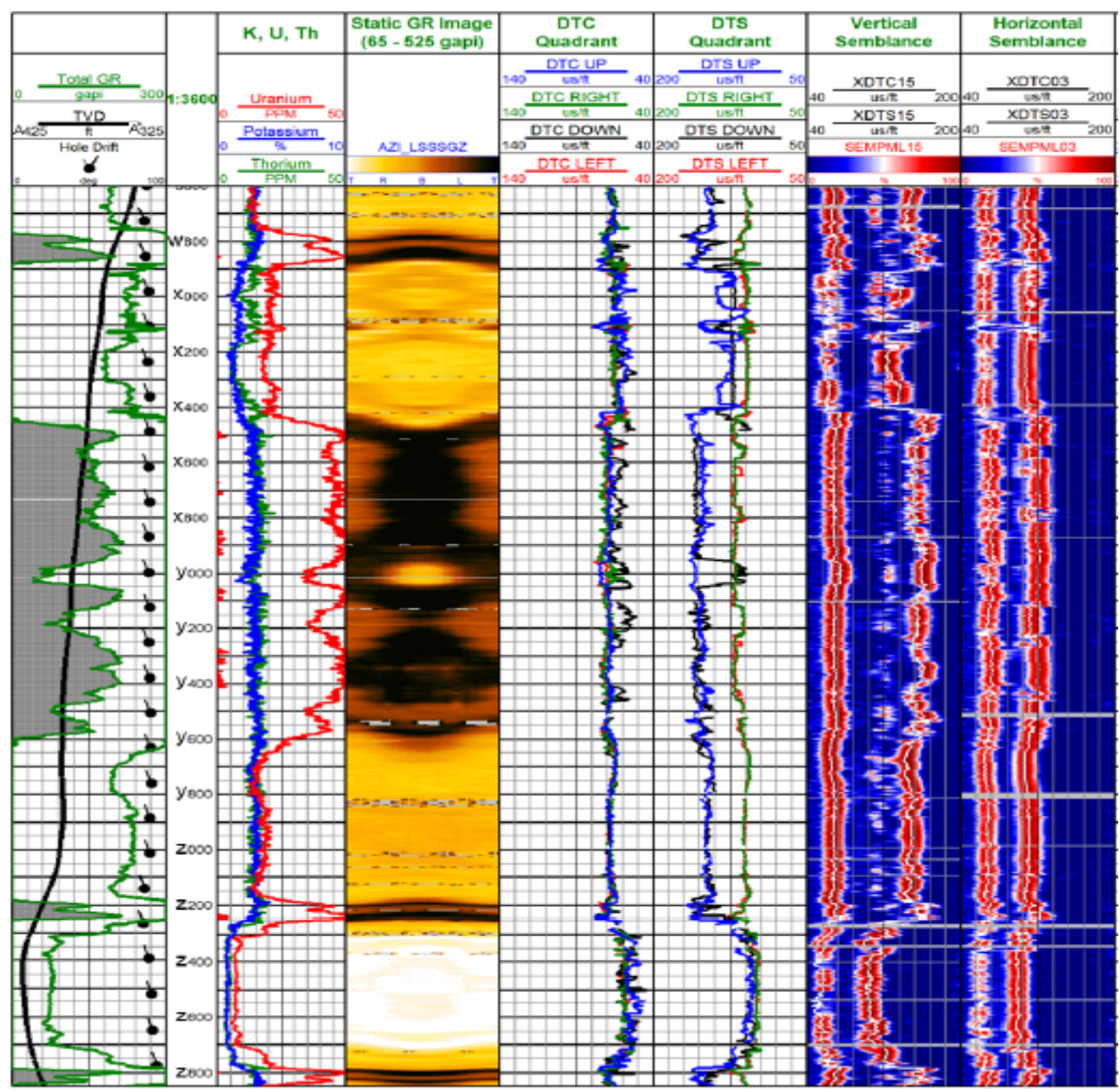

Figure 1.0: LWD Azimuthal Sonic and Spectral Gamma Ray signatures for a Marcellus shale well (Mickael, Barnet and Diab 2012)

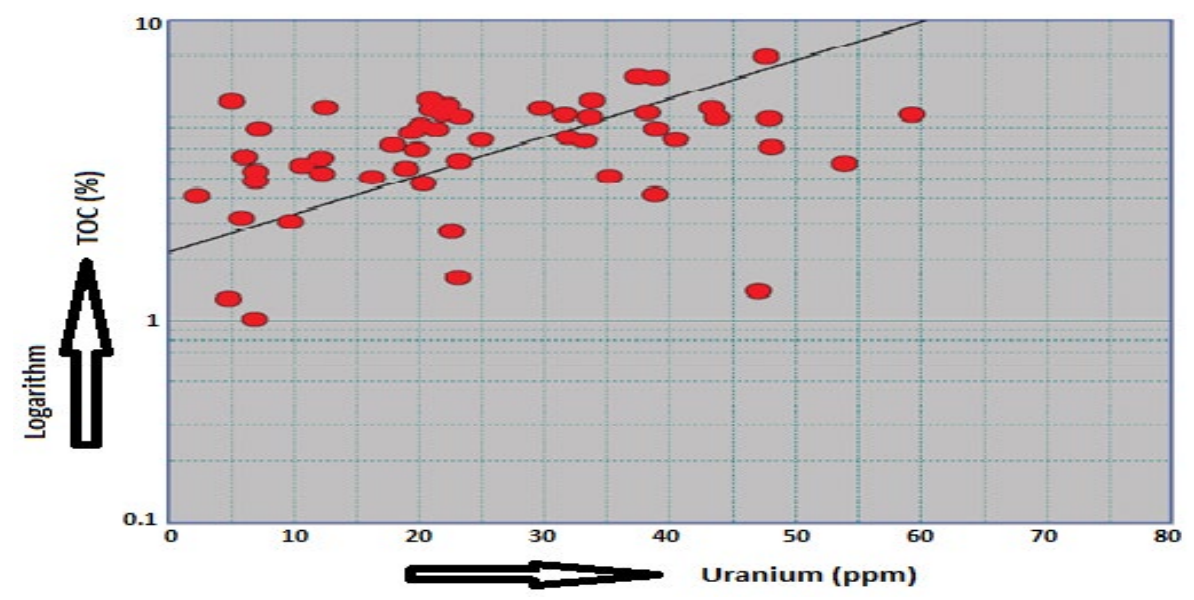

Figure 2.0: Cross plot of TOC against Uranium for typical marcellus shale reservoirs (Matt And Timothy 2010) 


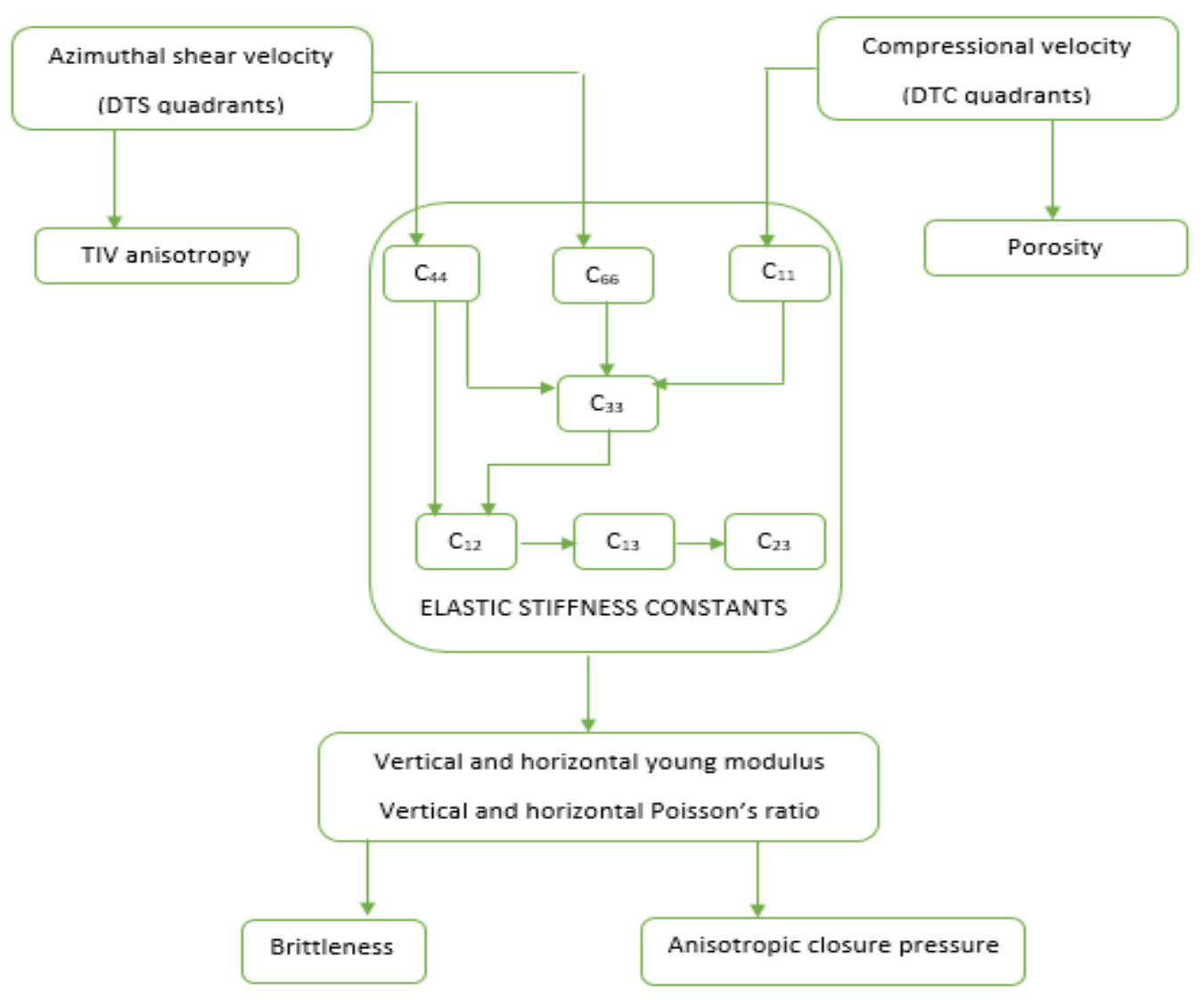

Figure 3.0: Sonic log workflow

\begin{tabular}{|c|c|}
\hline $\begin{array}{l}C_{11}=\rho V^{2} \text { horizontal compressional } \\
C_{14}=\rho V^{2}\end{array}$ & $C_{33}=C_{11}+2 C_{44}-2 C_{66}$ \\
\hline $\begin{array}{l}C_{55}=\rho V^{2} \text { fast vertical shear } \\
C_{66}=\rho V^{2}{ }_{\text {horizontally shear }}\end{array}$ & $C_{12}=C_{13}=C_{23}=C_{33}-2 C_{44}$ \\
\hline
\end{tabular}

Figure 4.0: Computation of elastic stiffness constants 


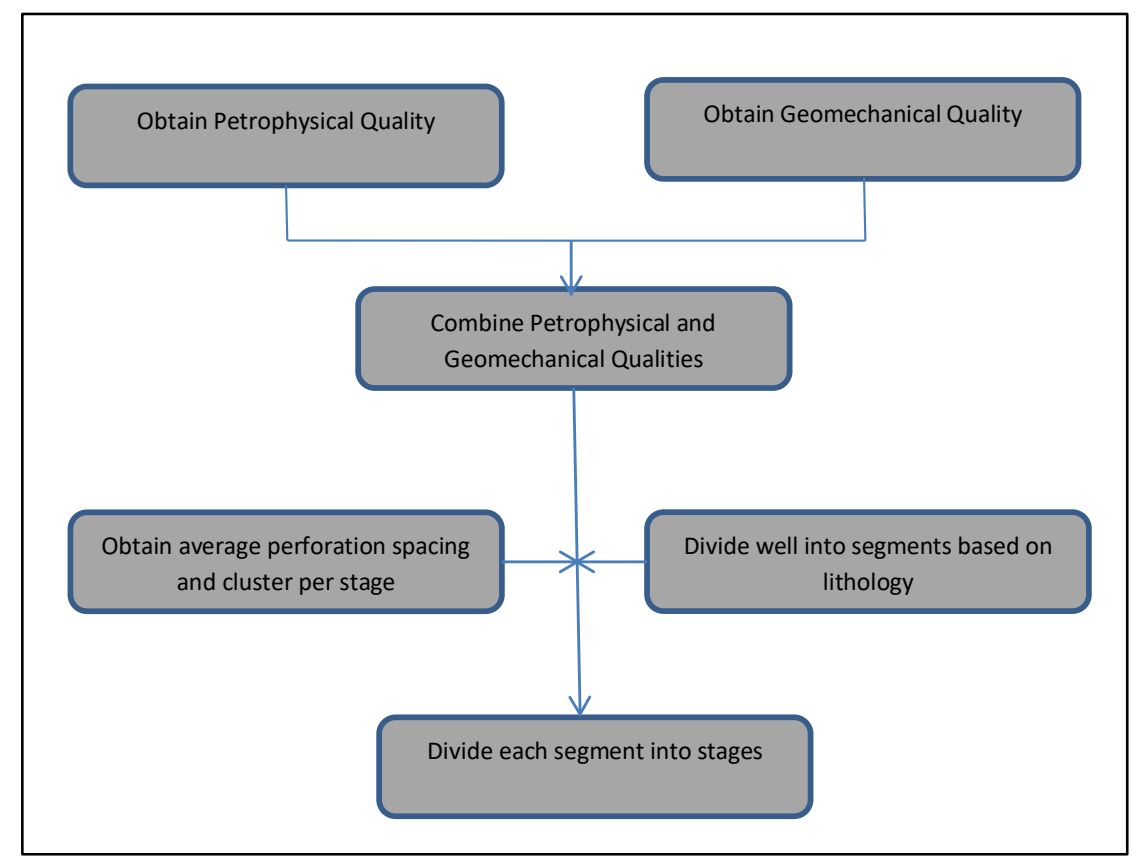

Figure 5.0: Interval selection algorithm

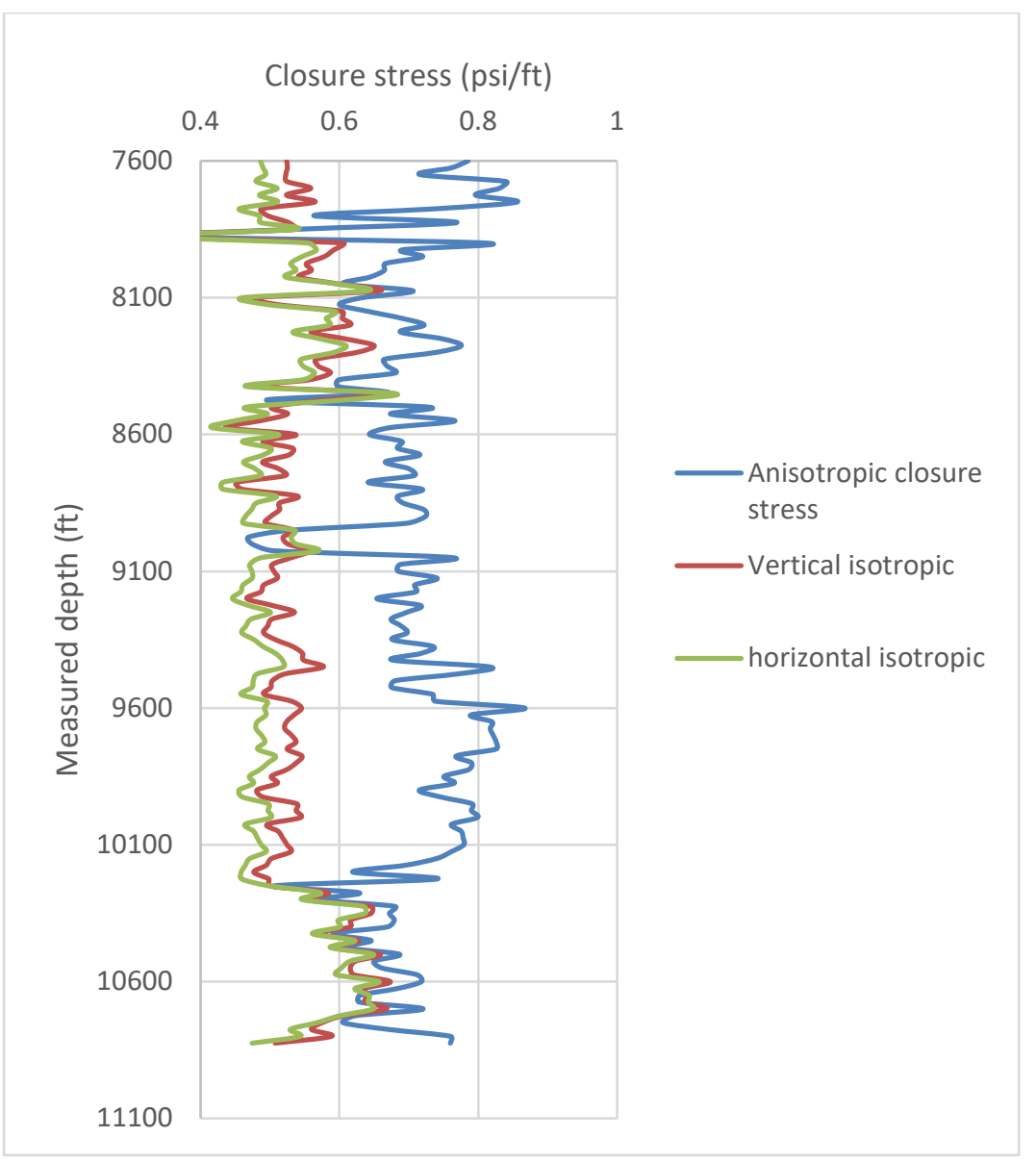

Figure 6.0: Anisotropic and isotropic closure stress signatures for the case study 


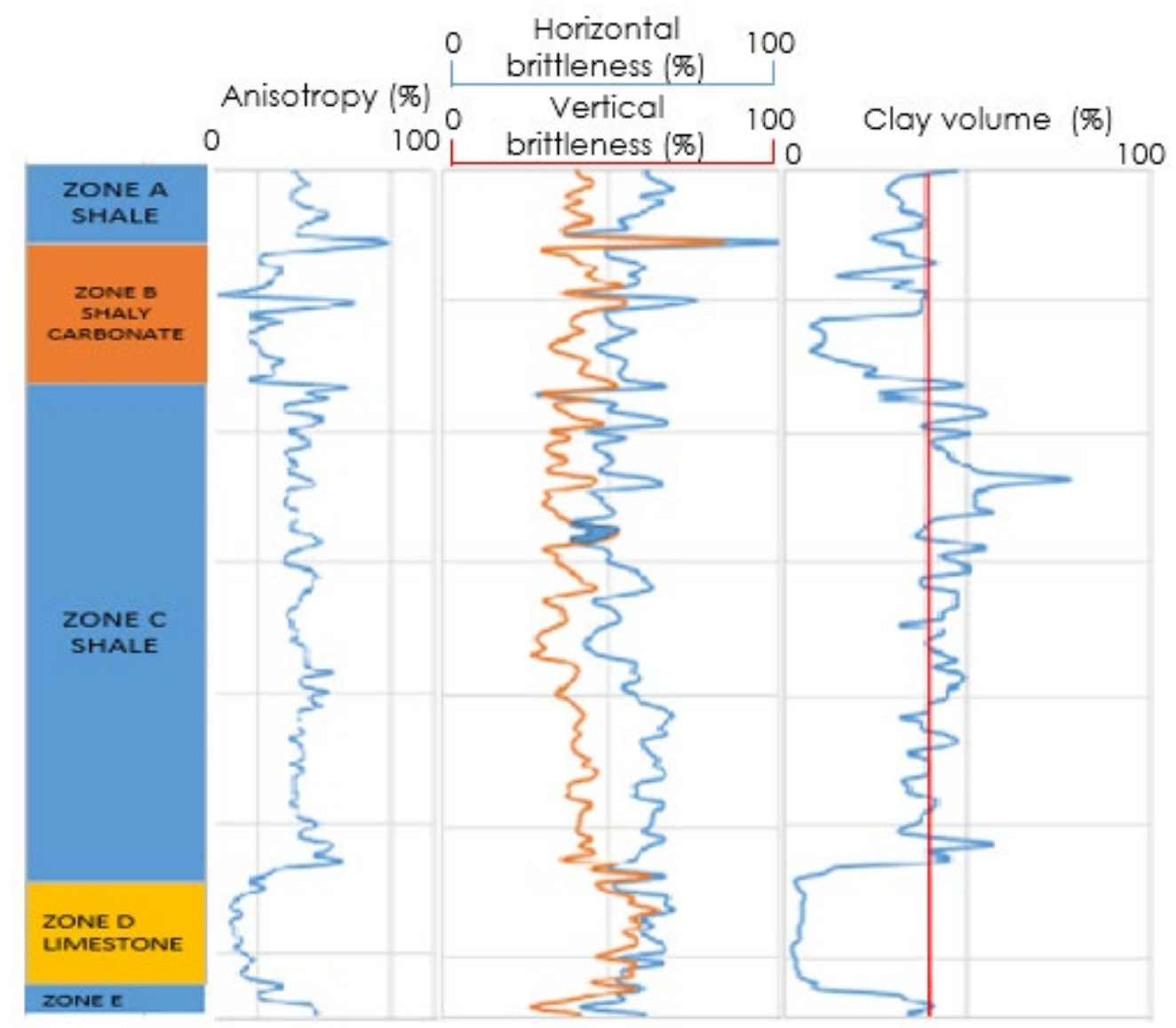

Figure 7.0: Anisotropy and Brittleness signatures for the case study 


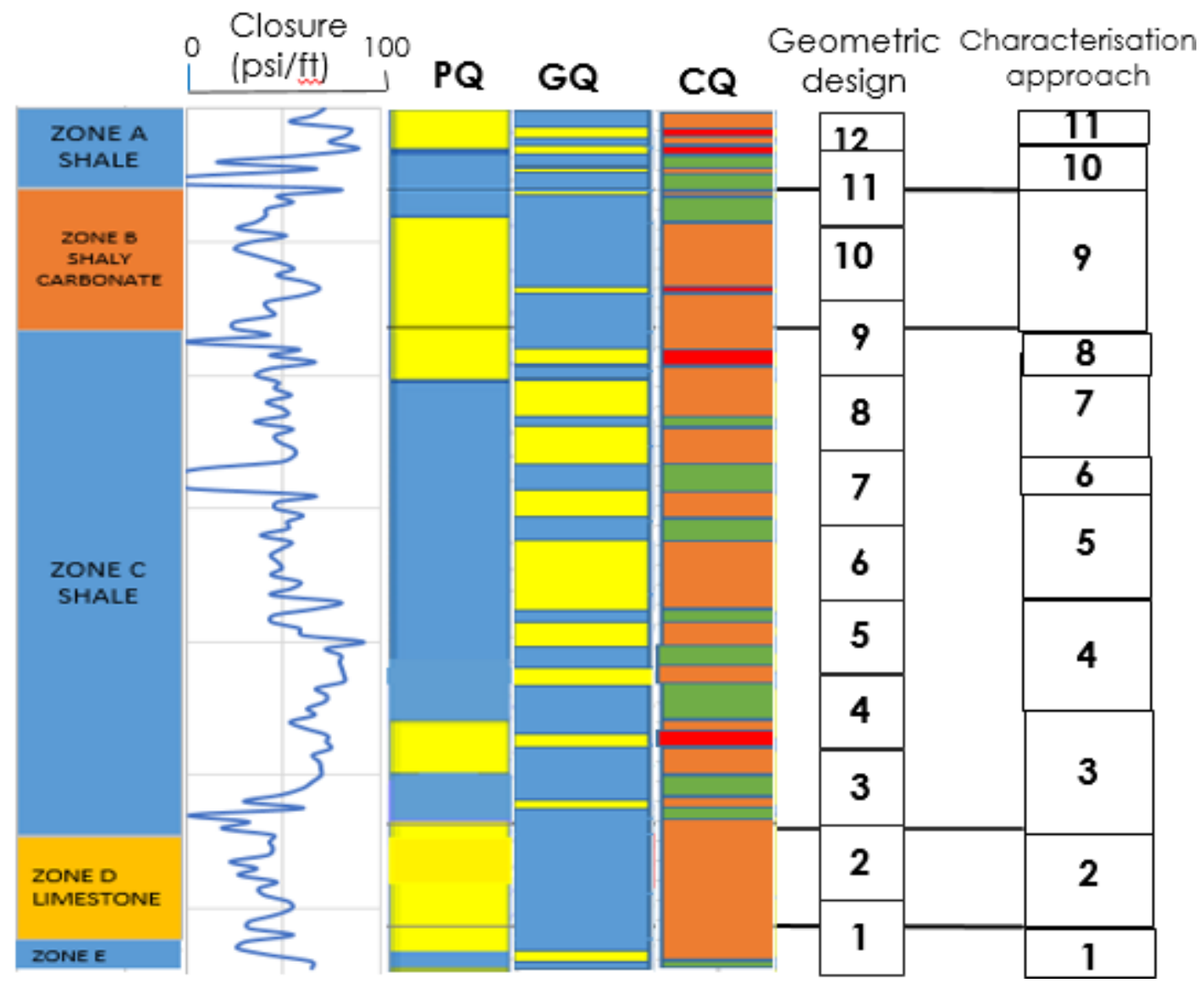

Figure 8.0: Comparison of stage placement using the conventional geometric approach and using the workflow in this study

\begin{tabular}{|c|c|}
\hline Colour code & Interpretation \\
\hline & Non-desirable \\
\hline & Desirable \\
\hline & Least suitable \\
\hline & Less suitable \\
\hline & Most suitable \\
\hline
\end{tabular}

Figure 9.0: Matrix for wellbore quality characterization 


\begin{tabular}{|c|c|}
\hline Property & Equations \\
\hline Poisson's ratio & $\mu_{v}=\frac{C_{13}}{\left(C_{11}+C_{12}\right)} \quad \mu_{h}=\frac{\left(C_{33} C_{12}-C_{13}\right)}{\left(C_{33} C_{11}-C_{13}\right)}$ \\
\hline young's modulus & $E_{v}=C_{33}-\frac{2 C_{13}{ }^{2}}{C_{11}+C_{12}} \quad E_{h}=\left(C_{11}-C_{12}\right) * \frac{\left(C_{11} C_{33}-2 C_{13}+C_{12} C_{33}\right)}{\left(C_{11} C_{33}-C_{13}{ }^{3}\right)}$ \\
\hline VTI Anisotropy & Anisotropy $(\%)=\frac{(\text { DTSmax }- \text { DTSmin }) * 100}{\text { DTS }_{\min }}$ \\
\hline brittleness & Brittleness $=\frac{[\text { BrittlenessE }+ \text { Brittlenessv }] * 100}{2}$ \\
\hline Isotropic closure stress & $\sigma_{\text {hiso }}=\frac{\mu}{1-\mu}\left(\sigma_{\mathrm{V}}-\mathrm{aPp}\right)+\mathrm{aPp}$ \\
\hline Anisotropic Closure stress & $\sigma_{\text {haniso }}=\frac{E_{h}}{E_{v}} \frac{\mu_{v}}{1-\mu_{h}}\left(\sigma_{v}-a P p\right)+a P p$ \\
\hline
\end{tabular}

